\title{
GMR
}

\section{Expression of the 2-dehydro-3-deoxyphosphooc- tonate aldolase $(K d s A)$ gene in mulberry leaves (Morus alba L.) is down-regulated under high salt and drought stress}

\author{
X.B. Yang*, S.L. Wu*, D.P. Zhu, H. Wu, T. Jiang, Y.H. Qian and F. Jiao \\ Mulberry Silk Institute of Animal Science and Technology College, \\ Northwest Science and Technology University, Yangling, China \\ *These authors contributed equally to this study. \\ Corresponding authors: Y.H. Qian / F. Jiao \\ E-mail: qyh@nwsuaf.edu.cn / fjiao@nwsuaf.edu.cn
}

Genet. Mol. Res. 14 (4): 11955-11964 (2015)

Received January 9, 2015

Accepted May 25, 2015

Published October 5, 2015

DOI http://dx.doi.org/10.4238/2015.October.5.9

ABSTRACT. The oligosaccharide 3-deoxy-D-manno-oct-2-ulosonic acid (Kdo) is a key component of lipopolysaccharide in Gramnegative bacteria, and is also part of the pectic polysaccharide rhamnogalacturonan (RG-II) of the plant cell wall. The enzyme KDO8-phosphate synthase (KDO8Ps), encoded by the 2-dehydro-3deoxyphosphooctonate aldolase $(K d s A)$ gene, catalyzes the first step in the synthesis of Kdo. In this study, the complete coding sequence of the $K d s A$ gene from mulberry leaves was cloned and the primary structure of KDO8Ps was deduced. Alignment of the amino acid sequence of KDO8Ps from mulberry with those of five other plant species revealed a high level of evolutionary conservation. A phylogenetic tree analysis demonstrated a short genetic distance among KDO8Ps proteins of different species. Expression of the $K d s A$ gene was higher in the second leaves than in the eighth leaves of mulberry, and was down-regulated under conditions of high salt or drought stress. Our results suggest that 
$K d s A$ expression is important for the growth of new plant tissues, and is sensitive to harsh environments.

Key words: Drought; High salt; KDO8Ps; KdsA gene expression; Mulberry

\section{INTRODUCTION}

3-Deoxy-D-manno-oct-2-ulosonic acid (Kdo) is an 8-carbon acidic sugar that bridges lipid A to lipopolysaccharide in all Gram-negative bacteria and is important for growth and division in these microorganisms (Raetz, 1990; Belunis et al., 1995). It has also has been found in plant cell walls as a component of the pectic polysaccharide rhamnogalacturonan (RG-II) (York et al., 1985; Séveno et al., 2010).

3-Deoxy-D-manno-octulosonate 8-phosphate synthase (KDO8Ps; EC 4.1.2.16), which is encoded by $K d s A$, catalyzes the condensation of phosphoenolpyruvate with D-arabinose-5phosphate to yield Kdo 8-phosphate (KDO8P) and inorganic phosphate, and plays a crucial role in the lipid A-Kdo biosynthesis pathway (Radaev et al., 2000; Delmas et al., 2008).

KDO8Ps is widely expressed in higher plants (Brabetz et al., 2000; Delmas et al., 2003); however, biochemical and structural investigations of the enzyme have largely been limited to bacteria (Woisetschlager and Hogenauer, 1987; Brabetz and Brade, 1997; Sarkar et al., 2012). The first evidence of a plant KDO8Ps was provided by the detection of Kdo-8-P synthase-like activities in eight different plant species, and from the partial purification of $K d s A$ and its characterization in spinach (Doong et al., 1991). Subsequently, Kdo-8-P synthase cDNA has been isolated from Pisum sativum (Brabetz et al., 2000), Solanum lycopersicum (Delmas et al., 2003), and Arabidopsis thaliana (Matsuura et al., 2003). Kdo-8-P synthase is encoded by a single-copy KdsA gene in tomato, but by two genes in Arabidopsis. In the latter, the homologous KDO8Ps genes AtKdsA1 (NM_202447) and AtKdsA2 (NM_001198082) share 93\% identity in their deduced protein sequences and are expressed mainly in shoots and roots (Matsuura et al., 2003). Generally, KDO8Ps sequences are well conserved among different species (Nelson et al., 2013), and the efficiency and substrate specificity of the Arabidopsis enzyme is similar to that of the Escherichia coli enzyme (Wu et al., 2004).

Mulberry trees are important as the food source of silkworms and for their environmental functions such as in drought relief and remediation of polluted water. In a previous study, we used two-dimensional electrophoresis and mass spectrometry analyses to show that mulberry KDO8Ps decreases under drought conditions (Feng et al., 2012). Here, we cloned the complete coding sequence region of the $K d s A$ gene from mulberry, and the expression profile of the $K d s A$ gene was compared in leaves of 11 mulberry cultivars. We found that expression of $K d s A$ in mulberry leaves was decreased by high salt and drought stress conditions.

\section{MATERIAL AND METHODS}

\section{Plant materials and treatment}

Eleven mulberry cultivars were used in this study: Pteris-Ichinose (PI, Japan); NeoIchinose (NI, Japan); Sweet-Mulberry No. 1 (SW1, Shaanxi, China, Fructus mori); White-Lotus (WL, Zhejiang, China); Shiping (SP, Shaanxi, China); Dashi (DS, Zhejiang, China, Fructus mori); Tongxiang-Green (TXG, Zhejiang, China); Local mulberry No. 401 (L401, Shaanxi, China); 
Yuanjiante (YJT, Japan, Morus mongolica); Red-Mulberry No. 1 (RM1, Shaanxi, China, Fructus mori); and Local mulberry No. 305 (L305, Shaanxi, China).

Fresh mulberry leaves were collected in the field from each cultivar and used for $K d s A$ gene cloning and the real-time quantitative PCR (qRT-PCR) analysis. In addition, the 2nd and the 8th leaves from the terminal buds of three cultivars (NI, L305, and RM1) were used for a comparison of young and old leaves. Six samples were collected from separate branches of each cultivar.

One-year-old $\mathrm{NI}$ trees were used to investigate the effects of drought and high salt treatment. The bare roots of $12 \mathrm{NI}$ trees were immersed in 5\% polyethylene glycol (PEG) 6000 Hoagland nutrient solution (the solution was refreshed every 2 days) and the roots of the control group were immersed in normal Hoagland nutrient solution. Mulberry leaves were collected from both groups on the 14th day. Twelve NI trees planted in plastic pots were used in high salt experiment. The matrix and mulberry tree in each pot was weighed (40-50 kg) firstly. $\mathrm{NaCl}(240-$ $300 \mathrm{~g}$ ) (equal to $0.6 \%$ of the matrix and mulberry tree) was dissolved in $1 \mathrm{~L}$ water and provided to each mulberry tree. Simultaneously the negative control group received an equivalent volume of pure water. Mulberry leaves were collected and analyzed after 5 days of treatment.

\section{RNA extraction and cDNA synthesis}

Total RNA was isolated from fresh mulberry leaves using Trizol reagent (Tiangen, Beijing, China) following the manufacturer instructions. The RNA concentration was determined using a spectrophotometer (Nanodrop, USA) and diluted to $300 \mathrm{ng} / \mu \mathrm{L}$. First strand cDNA synthesis was performed with the PrimeScript RT reagent kit (Takara, Kyoto, Japan).

\section{Cloning and sequence analysis of the $K d s A$ full-length cDNA}

A partial cDNA fragment of the $K d s A$ gene was obtained by PCR using the primers $\mathrm{P} 1$ and P2 (Table 1). PCR was carried out using a $20 \mu \mathrm{L}$ mixture and $2 X$ GoldStar Taq Mastermix (CWbiotech, China). PCR products were purified using a Gel-spin DNA extraction kit (Vigorous, China) following

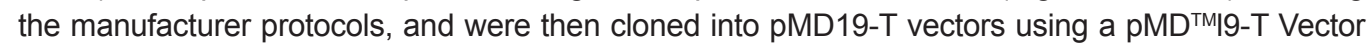
Cloning kit (Takara). The recombinant plasmid was transformed into E. coli TOP10 competent cells, which were smeared in solid LB medium and cultured at $37^{\circ} \mathrm{C}$ for $24 \mathrm{~h}$, then single colony was picked out and cultured in $5 \mathrm{~mL}$ liquid LB medium for another $24 \mathrm{~h}$ prior to extraction; recombinant plasmids were positively identified and sequenced using GeneScript (Nanjing, China). Primers for 3' rapid amplification of cDNA ends (RACE) and 5' RACE were designed using known sequences.

For 3' RACE PCR, RNA was reverse transcribed into cDNA with primer P3 using Reverse Transcriptase M-MLV (RNase H-) kit (Takara). P4 and 3' Gsp1 were used in the first round PCR, while P4 and 3' Gsp2 were used in the second round PCR. For 5' RACE PCR, the specific primer used for reverse transcription was P5' R. P5 and 5' Gsp1, P4 and 5' Gsp2 were used for the nested PCR in the first and second rounds, respectively. The sequencing process was performed by GeneScript (Nanjing, China) as described previously.

\section{Bioinformatic analysis}

The open reading frame (ORF) of the full-length $K d s A$ cDNA sequence was predicted 
using Bioxm2.6 and the protein sequence were deduced. The MEGA5.10 software was used for homology analysis and phylogenetic tree construction.

\begin{tabular}{lll}
\multicolumn{2}{l}{ Table 1. Primers used in this study. } \\
\hline Primer names & Primer sequences 5' to 3' & Sequence information \\
\hline P1 & GGTCCHAATGTGATTGAATC & Degenerate primer F \\
P2 & AAGRCCTCCACTKGCAACAC & Degenerate primer R \\
P3 & GGCCACGCGTCGACTAGTACTTTTTTTTTTTTTTTTT & For 3' reverse transcription \\
P4 & GGCCACGCGTCGACTAGTAC & For 1st round 3GTCGACTAGTAConTTTTTTTTed PCR \\
P5 & GGCCACGCGTCGACTAGTACGGGGGGGGG & For 1st round 5GTCGACTAGT \\
3'Gsp1 & GTGCTCCTTCTGTAATGGTA & For 1st round 3TCTGTAATGG \\
3'Gsp2 & GACATTACGCATTCACTACAAC & For 2nd round 3' RACE PCR \\
P5'R & GATGTTCTGTTGGCTTTGTC & For 5' reverse transcription \\
5'Gsp1 & TACCATTACAGAAGGAGCAC & For 1st round 5CAGAAGGAGC \\
5'Gsp2 & GTTGTAGTGAATGCGTAATGTC & For 2nd round 5' RACE PCR \\
M13F & AGGGTTTTCCCAGTCACG & For sequencing \\
M13R & GAGCGGATAACAATTTCACAC & For sequencing \\
\hline
\end{tabular}

\section{mRNA expression levels in mulberry cultivars}

The expression pattern of $K d s A$ in 11 mulberry cultivars was determined by qRT-PCR. Differences in expression profiles among young and old leaves, and between control leaves and those subjected to conditions of high salt and drought stress were investigated using the same method. The primers used for analysis of KdsA expression were F, 5'-GTGCTCCTTCTGTAATGGTA-3', and R, 5'-GTTGTAGTGAATGCGTAATGTC-3'. Morus alba ACT2 (GenBank accession No. HQ163774.1) was selected as the endogenous reference gene for calibration using the following primers: ACT2-F, 5'-GTGGCCGTACAACTGGTATT-3', and ACT2-R, 5'-GCCAGGTCAAGACGAAGAAT-3'.

qRT-PCR was performed using a Bio-Rad iQ5 instrument (Bio-Rad, USA) and SYBR Green Real-time PCR Master mix (Takara) in a $20-\mu \mathrm{L}$ reaction mixture containing $1 \mu \mathrm{L}$ template cDNA. The qRT-PCR protocol consisted of a two-step method: $95^{\circ} \mathrm{C}$ for $3 \mathrm{~min}$, followed by 40 cycles of $95^{\circ} \mathrm{C}$ for $5 \mathrm{~s}$ and $60^{\circ} \mathrm{C}$ for $30 \mathrm{~s}$. Melting curves were determined with $0.5^{\circ} \mathrm{C}$ increments from $55^{\circ}$ to $95^{\circ} \mathrm{C}$. The relative mRNA expression level was determined by the $2^{-\Delta \Delta \mathrm{Ct}}$ method (Livak and Schmittgen, 2001).

\section{Statistical analysis}

SPSS 19.0 was used to analyze the qRT-PCR data analysis. The Kruskal-Wallis test was performed to detect differences among the cultivars, and the Wilcoxon test was used to determine differences between the 2nd and the 8th leaves, as well as between the treatment groups and the control group.

\section{RESULTS}

\section{Sequence of the $K d s A$ gene and the deduced protein}

The full-length $K d s A$ cDNA was $1350 \mathrm{bp}$, including a 249-bp 5'-untranslated region (UTR), an 873-bp ORF, a 231-bp 3'-UTR (Figure 1), and the typical AATAA motif. The sequence has been submitted to the GenBank database as accession No. KM588119. The primary structure of 
the predicted protein is shown in Figure 1. The ORF encoded 290 amino acids with a predicted molecular weight of $31.57 \mathrm{kDa}$ and an isoelectric point of 6.60 .

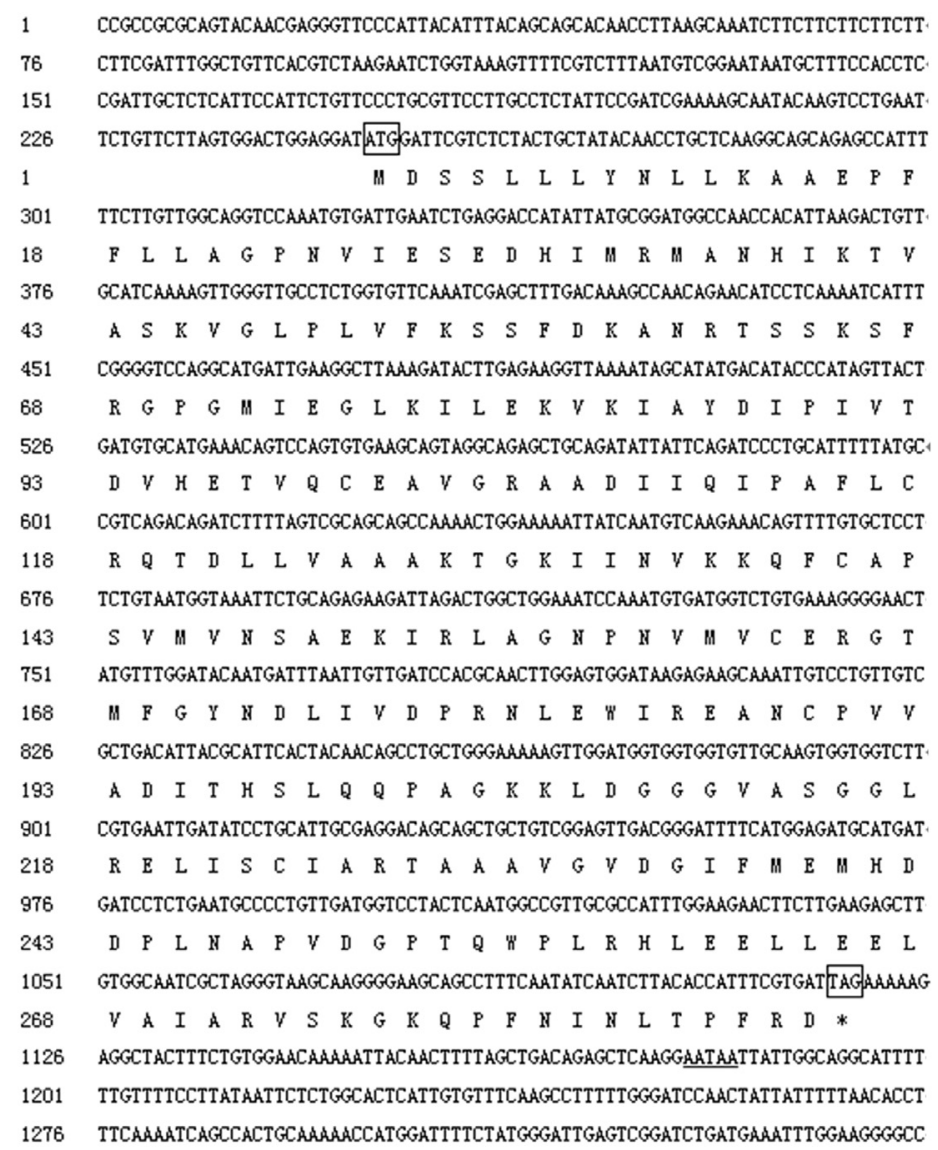

Figure 1. Nucleotide sequence of the KdsA cDNA and the predicted primary amino acid sequence of the KDO8Ps protein.

\section{Sequence homology and phylogenetic analysis of the KDO8Ps protein}

Alignment of the mulberry KDO8Ps sequence with those of 5 other plant species (Figure 2) showed similar total lengths and high homology of the amino acids, indicating a conserved overall structure. Mulberry KDO8Ps showed high sequence identities with that of Cucumis sativus (XP004171872; 90\%), A. thaliana (NP001031300; 88\%), Prunus mume (XP008226054; 89\%), Fragaria vesca subsp (XP004306551; 88\%), and Cucumis melo (XP008457489; 88\%).

A phylogenetic tree analysis of KDO8Ps proteins showed that mulberry KDO8Ps shared the highest identity with Citrus sinensis (XP006493775), and greater similarity with proteins from other plant species compared to bacterial and animal species (Figure 3). Nevertheless, $42 \%$ identity was found between mulberry and E. coli DH1 (ACX40072), which suggested evolutionary conservation of the Kdo biosynthesis pathway. The ${ }^{113} \mathrm{PAFLxR}{ }^{118}$ motif appears conserved in many 
species and provides a key contact for the D-arabinose 5-phosphate substrate; mutant proteins that have substitutions in this motif demonstrate poor catalytic function (Allison et al., 2013).

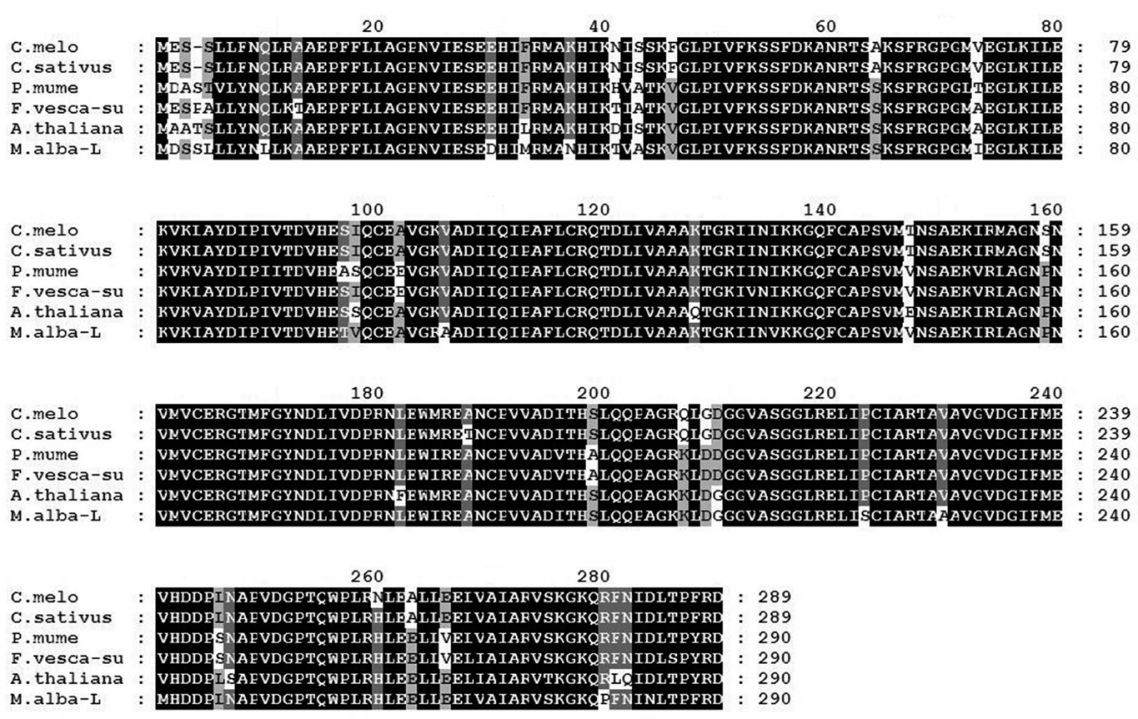

Figure 2. Multiple sequence alignment of KDO8Ps with homologous proteins. Identical sequences are shown in black, while letters in white or gray mean not or partly identical.

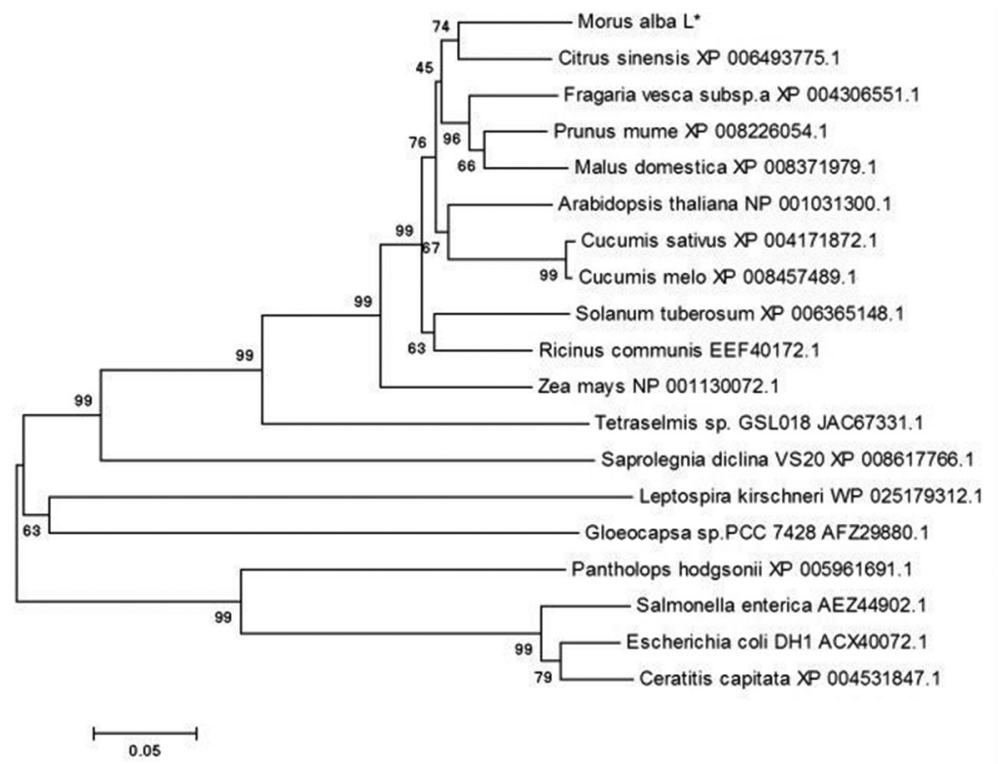

Figure 3. Phylogenetic tree of KDO8Ps based on protein sequences. The genetic distances were calculated using the $\mathrm{p}$-distance method. The percentages of replicate tree in which the associated taxa clustered together are shown next to the branches (the bootstrap test was 10,000 replicates). The GenBank accession numbers are listed after the species names. The tree was drawn to scale, with branch lengths in the same units as those of the evolutionary distances used to infer the phylogenetic tree. 


\section{Expression of $K d s A$ under normal conditions and after drought or high salt stress}

qRT-PCR was used to determine the expression profile of the $K d s A$ gene in 11 mulberry cultivars. Standard curves were established to determine the PCR efficiency of $K d s A$ and Act2 gene amplification (1.06 and 1.04, respectively). The 8th leaves of L401 and YJT cultivars had a relatively high level of KdsA expression compared to TXG, NI, RM1 and L305 cultivars (Figure 4); the remaining cultivars showed similar, intermediate levels of expression. The three cultivars tested, NI, RM1, and L305, showed significantly lower levels of KdsA expression in the 2nd leaves compared to the 8th leaves (Figure 5). Possibly, new tissue growth requires higher Kdo expression levels for the formation of the RG-II structure, which plays a major physiological role in the cell wall (Delmas et al., 2008). Furthermore, new leaves require more KDO8Ps to maintain the high efficiency of the Kdo biosynthesis pathway. We found that the level of $K d s A$ expression was significantly lower in plants exposed to either salt or drought stress (Figure 6). The reason for the decline in $K d s A$ expression under conditions of high salt and drought stress may also attributed to the Kdo biosynthesis pathway (Figure 6 ), with the down-regulated $K d s A$ gene expression reflecting the decreased KDO8Ps activity. Physiological regulation is required to meet the basic needs of plant survival under harsh environmental conditions; therefore, the rate of new tissue growth and development is reduced.

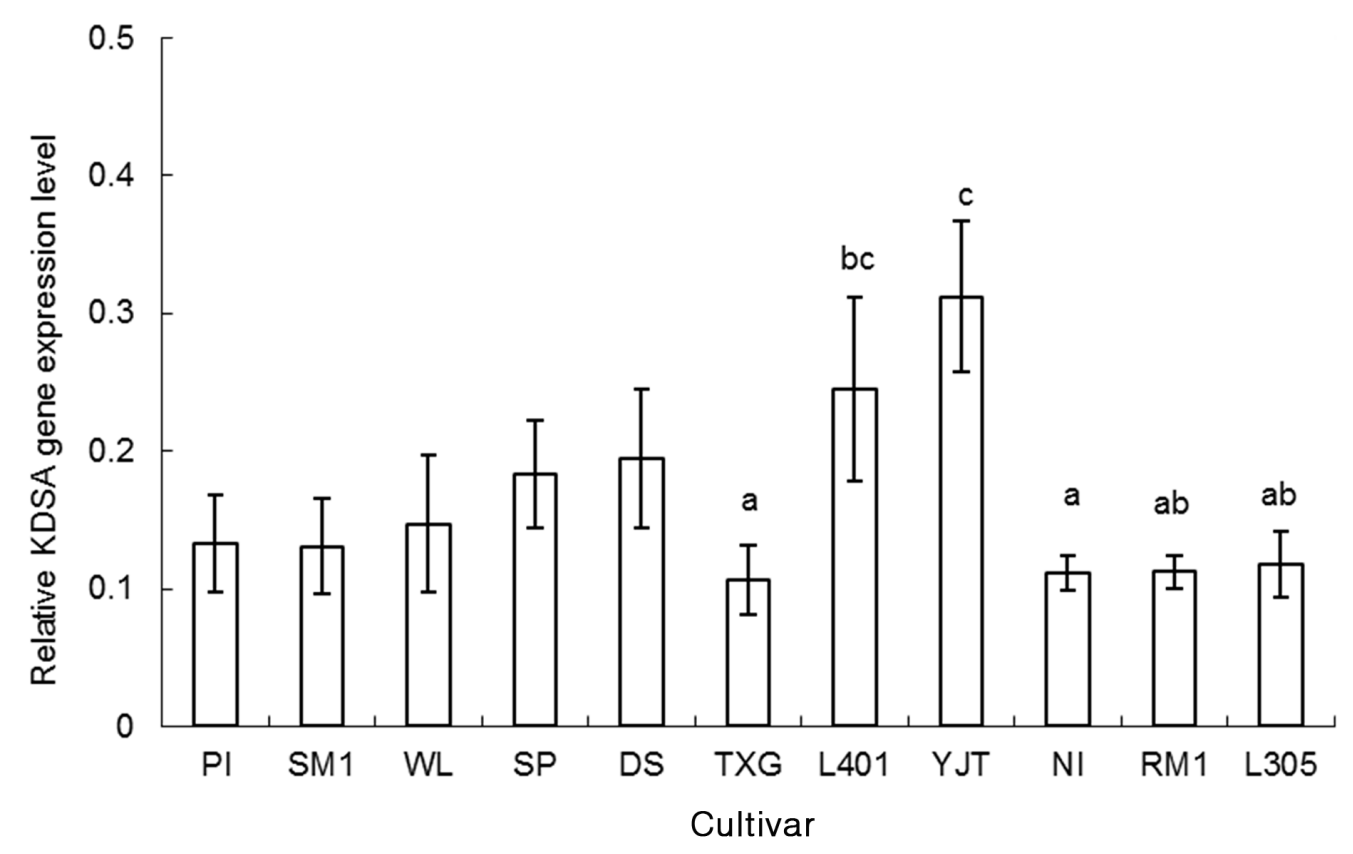

Figure 4. Expression profiles of $K d s A$ in the 8th leaves of 11 mulberry cultivars (see "Material and methods"). KruskalWallis tests were performed and significant differences are indicated by different letters; the same letter or no letter indicates no significant difference between two cultivars. 


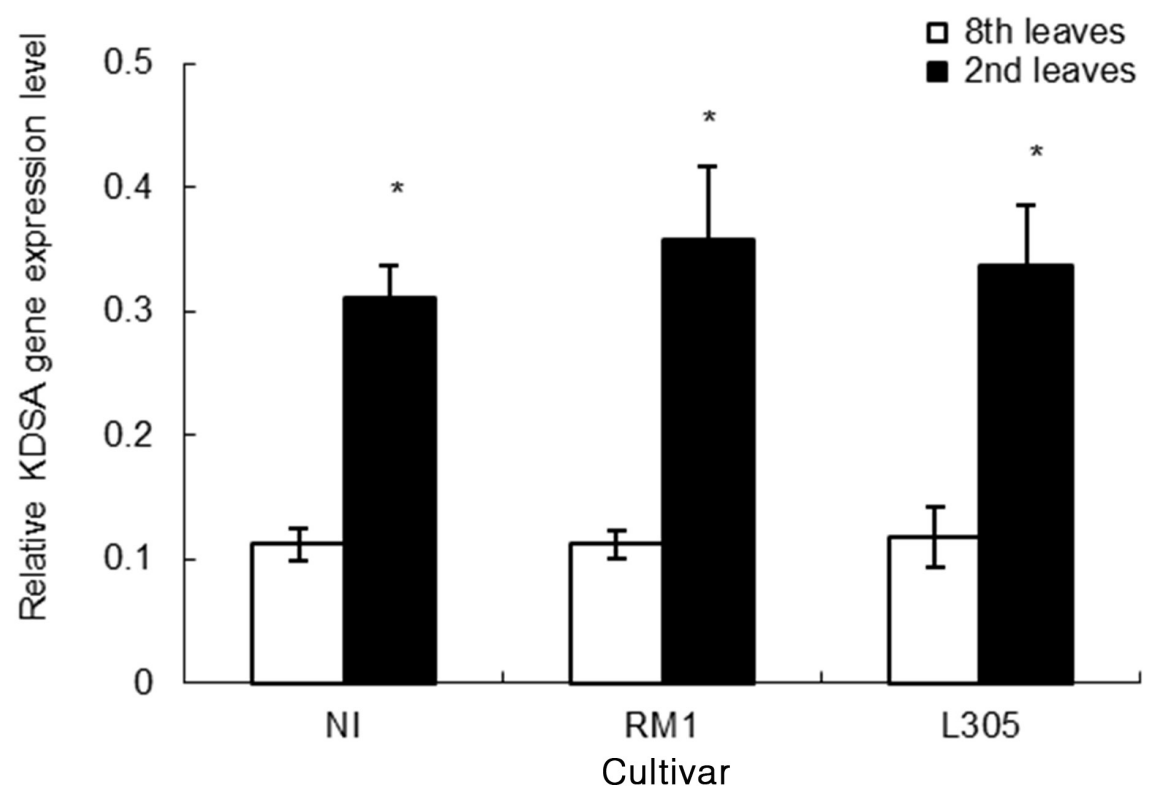

Figure 5. Comparison of $K d s A$ expression in 8 th and 2 nd leaves of three mulberry cultivars selected. The Wilcoxon test was used to detect significant differences: * $P<0.05$.

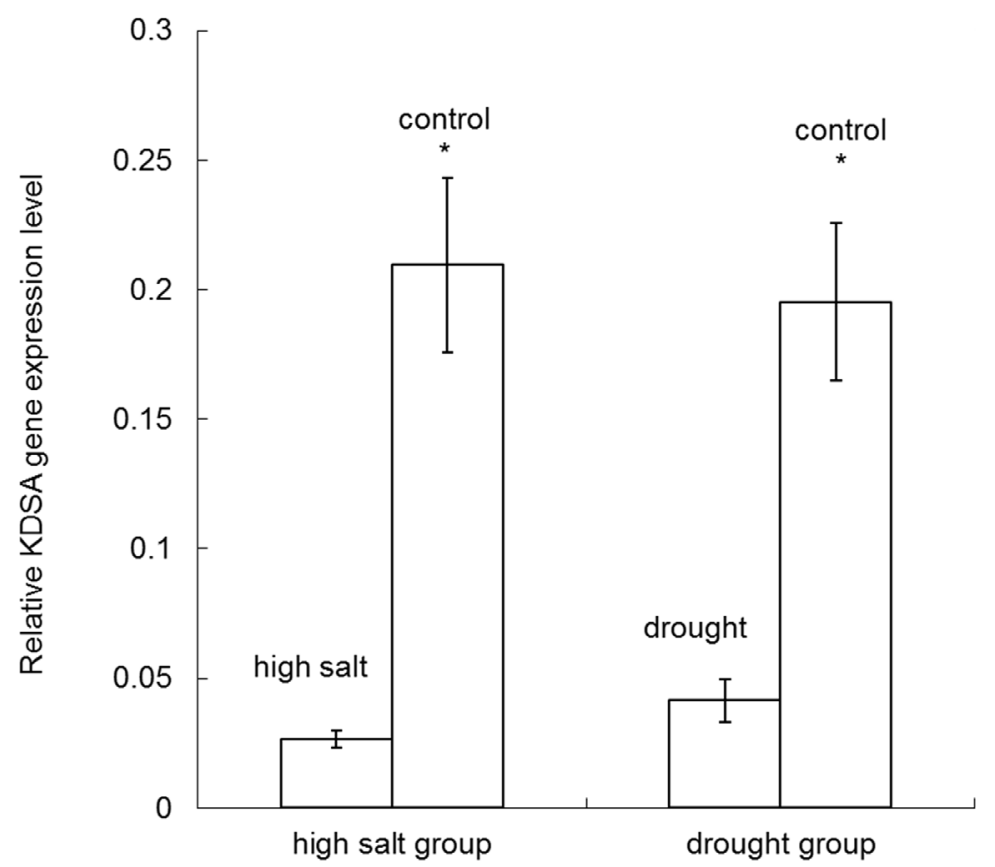

Figure 6. Differential expression of the $K d s A$ gene under conditions of high salt or drought stress. The Wilcoxon test was performed to determine the differences between treatment and the control group. ${ }^{*} \mathrm{P}<0.05$. 


\section{DISCUSSION}

The Kdo biosynthesis and activation pathway shows considerable conservation across a wide evolutionary spectrum from plants to bacteria, although Kdo may be incorporated into very different macromolecules (Smyth and Marchant, 2013). Kinetic studies have shown that the KDO8Ps enzymes of Arabidopsis and E. coli catalyze the condensation of D-arabinose 5-phosphate and phosphoenolpyruvate to generate Kdo-8-P with similar efficiencies ( $k_{\text {cat }}, 5.9 \mathrm{~s}^{-1}$ and $6.8 \mathrm{~s}^{-1}$, respectively) (Wu et al., 2004). KDO8Ps of $P$. sativum can functionally complement a KdsA mutant of the Gram-negative bacterium Salmonella enterica (Brabetz et al., 2000). Here, we confirmed that mulberry KDO8Ps shares high amino acid sequence identity with other plant species, and even with those of animals and bacteria.

The AtKdsA2 gene promoter is preferentially induced in young tissues such as cotyledons, primary leaves of the shoot, style and anthers of mature flowers, and hydathodes (Delmas et al., 2008). This effect was confirmed in this study by our finding that $K d s A$ expression was higher in the 2nd leaves than in the 8th leaves. Similar results have been reported elsewhere showing the preferential expression of the LeKdsA gene in dividing tissues and organs in the tomato (Delmas et al., 2003). Furthermore, AtKdsA2 is also expressed in reproductive tissues (Iwai et al., 2006).

The sugar Kdo of the RG-II structure in the cell wall is vital for plant growth. Kdo is linked to the RG-II GalA backbone and participates in the formation of the side chain C, which is composed of the a-D-Kdo-a-L-Rhap disaccharide (O'Neill et al., 2004); therefore, impairment in the synthesis of Kdo results in the absence of side chain C from RG-II. KDO8Ps catalyzes the first step in the biosynthesis of Kdo and is an essential enzyme in Gram-negative enteric bacteria for maintaining bacterial viability (Ray, 1980; Luke et al., 2003). The production of Kdo in the cytosol is very highly correlated with the level of $K d s A$ gene transcription. In our previous study, we showed that KDO8Ps was almost completely lost under conditions of drought stress (Feng et al., 2012). In the current study, both high salt and drought stresses directly or indirectly decreased $K d s A$ expression, which may result in a presumed subsequent decline in the catalytic efficiency of KDO8Ps and a concomitant reduction in Kdo biosynthesis. Such changes in enzyme activity might prevent the construction of new cell walls. Thus, we conclude that regulation of $K d s A$ gene expression represents one step in the complicated mechanisms by which plants adjust to harsh environments.

\section{CONCLUSION}

High identity shared by the mulberry KDO8Ps amino acid sequence with five other plant species indicated evolutionary conservation of the plant Kdo biosynthesis pathway. Higher transcription of the $K d s A$ gene in new leaves compared to old leaves suggested an important role of KDO8Ps in new plant tissues formation, in accordance with previous research. In this study, based on the decreased $K d s A$ gene expression under high salt and drought stresses, it suggested that $K d s A$ gene expression is sensitive to harsh environments.

\section{ACKNOWLEDGMENTS}

Research supported by the Modern Agricultural Technology System (\#CARS-35), the Public Service Sectors (Agriculture) Special (\#201303057), and the Fundamental Research Special of Northwest A\&F University (\#ZD2012008 and \#XNY2013-27). 


\section{REFERENCES}

Allison TM, Cochrane FC, Jameson GB and Parker EJ (2013). Examining the role of intersubunit contacts in catalysis by 3-deoxy-D-manno-octulosonate 8-phosphate synthase. Biochemistry 52: 4676-4686.

Belunis CJ, Clementz T, Carty SM and Raetz CRH (1995). Inhibition of lipopolysaccharide biosynthesis and cell growth following inactivation of the kdtA gene in Escherichia coli. J. Biol. Chem. 270: 27646-27652.

Brabetz W and Brade $\mathrm{H}$ (1997). Molecular cloning, sequence analysis and functional characterization of the gene $k d s A$, encoding 3-deoxy-D-manno-2-octulosonate-8-phosphate synthase of Chlamydia psittaci 6BC. Eur. J. Biochem. 244: 66-73.

Brabetz W, Wolter FP and Brade H (2000). A cDNA encoding 3-deoxy-D-manno-oct-2-ulosonate-8-phosphate synthase of Pisum sativum L. (pea) functionally complements a $k d s A$ mutant of the Gram-negative bacterium Salmonella enterica. Planta 212: 136-143.

Delmas F, Petit J, Joubès J, Séveno M, et al. (2003). The gene expression and enzyme activity of plant 3-deoxy-D-manno-2octulosonic acid-8-phosphate synthase are preferentially associated with cell division in a cell cycle-dependent manner. Plant Physiol. 133: 348-360.

Delmas F, Séveno M, Northey JG, Hernould M, et al. (2008). The synthesis of the rhamnogalacturonan II component 3-deoxyD-manno-2-octulosonic acid (Kdo) is required for pollen tube growth and elongation. J. Exp. Bot. 59: 2639-2647.

Doong R, Ahmad S and Jensen R (1991). Higher plants express 3-deoxy-D-manno-octulosonate-8-phosphate synthase. Plant Cell Environm. 14: 113-120.

Feng J, Lu-Lu P, Sha H, Xin-Hua S, et al. (2012). Analysis on differential expression of proteins in mulberry leaves under drought stress. Sci. Sericult. 6: 968-974.

Iwai H, Hokura A, Oishi M, Chida H, et al. (2006). The gene responsible for borate cross-linking of pectin rhamnogalacturonan-II is required for plant reproductive tissue development and fertilization. Proc. Natl. Acad. Sci. U. S. A. 103: 16592-16597.

Livak KJ and Schmittgen TD (2001). Analysis of relative gene expression data using real-time quantitative PCR and the $2^{\Delta \Delta c t}$ method. Methods 25: 402-408.

Luke NR, Allen S, Gibson BW and Campagnari AA (2003). Identification of a 3-deoxy-D-manno-octulosonic acid biosynthetic operon in Moraxella catarrhalis and analysis of a KdsA-deficient isogenic mutant. Infect. Immun. 71: 6426-6434.

Matsuura K, Miyagawa I, Kobayashi M, Ohta D, et al. (2003). Arabidopsis 3-deoxy-d-manno-oct-2-ulosonate-8-phosphate synthase: cDNA cloning and expression analyses. J. Exp. Bot. 54: 1785-1787.

Nelson SK, Kelleher A, Robinson G, Reiling S, et al. (2013). Structure of 2-keto-3-deoxy-D-manno-octulosonate-8-phosphate synthase from Pseudomonas aeruginosa. Acta Crystallogr. Sect. F Struct. Biol. Cryst. Commun. 69: 1084-1088.

O'Neill MA, Ishii T, Albersheim P and Darvill AG (2004). Rhamnogalacturonan II: structure and function of a borate cross-linked cell wall pectic polysaccharide. Annu. Rev. Plant Biol. 55: 109-139.

Radaev S, Dastidar P, Patel M, Woodard RW, et al. (2000). Structure and mechanism of 3-deoxy-D-manno-octulosonate 8-phosphate synthase. J. Biol. Chem. 275: 9476-9484.

Raetz CRH (1990). Biochemistry of endotoxins. Annu. Rev. Biochem. 59: 129-170.

Ray PH (1980). Purification and characterization of 3-deoxy-D-manno-octulosonate 8-phosphate synthetase from Escherichia coli. J. Bacteriol. 141: 635-644.

Séveno M, Séveno-Carpentier E, Voxeur A, Menu-Bouaouiche L, et al. (2010). Characterization of a putative 3-deoxy-Dmanno-2-octulosonic acid (Kdo) transferase gene from Arabidopsis thaliana. Glycobiology 20: 617-628.

Sarkar M, Maganti L, Ghoshal N and Dutta C (2012). In silico quest for putative drug targets in Helicobacter pylori HPAG1: molecular modeling of candidate enzymes from lipopolysaccharide biosynthesis pathway. J. Mol. Model. 18: 1855-1866.

Smyth KM and Marchant A (2013). Conservation of the 2-keto-3-deoxymanno-octulosonic acid (Kdo) biosynthesis pathway between plants and bacteria. Carbohydr. Res. 380: 70-75.

Woisetschlager M and Hogenauer G (1987). The kdsA gene coding for 3-deoxy-D-manno-octulosonic acid 8-phosphate synthetase is part of an operon in Escherichia coli. Mol. Gen. Genet. 207: 369-373.

Wu J, Patel M, Sundaram A and Woodard R (2004). Functional and biochemical characterization of a recombinant Arabidopsis thaliana 3-deoxy-D-manno-octulosonate 8-phosphate synthase. Biochem. J. 381: 185-193.

York WS, Darvill AG, McNeil M and Albersheim P (1985). 3-deoxy-D-manno-2-octulosonic acid (KDO) is a component of rhamnogalacturonan II, a pectic polysaccharide in the primary cell walls of plants. Carbohydr. Res. 138: 109-126. 